\title{
Synchronized Neuronal Discharge in the Basal Ganglia of Parkinsonian Patients Is Limited to Oscillatory Activity
}

\author{
Ron Levy, ${ }^{1}$ William D. Hutchison, ${ }^{1,2,3}$ Andres M. Lozano,, ${ }^{2,3}$ and Jonathan O. Dostrovsky ${ }^{1,3}$ \\ ${ }^{1}$ Department of Physiology, Faculty of Medicine, University of Toronto, Toronto, Ontario, Canada M5S 1A8, 2 Division of \\ Neurosurgery, Department of Surgery, University of Toronto, The Toronto Western Hospital, Toronto, Ontario, \\ Canada M5T 2S8, and ${ }^{3}$ The Toronto Western Research Institute, The Toronto Western Hospital, Toronto, Ontario, \\ Canada M5T 2S8
}

It has been proposed that an increase in synchronization between neurons in the basal ganglia contributes to the clinical features of Parkinson's disease (PD). To examine this hypothesis, we looked for correlations in the discharge activity of pairs of neurons in the globus pallidus internus (GPi), globus pallidus externus (GPe), and the substantia nigra pars reticulata (SNr). Recordings were performed in PD patients undergoing functional stereotactic mapping for pallidotomy (eight patients) or subthalamic nucleus deep brain stimulation (four patients). A double-microelectrode setup was used to simultaneously record from neurons separated by distances as small as 250 $\mu \mathrm{m}$. In the five pallidotomy patients without limb tremor during the procedure, none of the $73 \mathrm{GPi}$ pairs and $15 \mathrm{GPe}$ pairs displayed synchronous activity. In the three pallidotomy pa- tients with limb tremor, 6 of 21 GPi pairs and 5 of 29 GPe pairs displayed oscillatory synchronization in the frequency range of the ongoing limb tremor $(3-6 \mathrm{~Hz})$ or at higher frequencies (15-30 Hz). Synchronized activity was not observed in the SNr (10 pairs). The findings indicate that oscillatory synchronization between pairs of GPi or GPe neurons is found in patients with limb tremor. These results also suggest that overt neuronal synchronization, which may be attributable to an increase in direct synaptic connections or common collateral afferent inputs, is not present in the basal ganglia of patients with PD.

Key words: Parkinson's disease; globus pallidus; subthalamic nucleus; substantia nigra pars reticulata; synchronization; limb tremor
Akinesia, limb tremor, and rigidity are the main motor symptoms of Parkinson's disease (PD) (Lang and Lozano, 1998) and have been associated with an excessive inhibition of thalamic motor and brainstem nuclei by the globus pallidus internus (GPi), the major output nucleus of the basal ganglia (Crossman et al., 1985; Miller and DeLong, 1987; Albin et al., 1989; DeLong, 1990; Eidelberg et al., 1997). In addition to an augmentation of GPi firing rates, monkeys rendered parkinsonian with 1-methyl-4phenyl-1,2,3,6-tetrahydropyridine (MPTP) display an increase in the bursting activity of GPi, globus pallidus externus (GPe), and substantia nigra pars reticulata $(\mathrm{SNr})$ neurons (Miller and DeLong, 1987; Filion and Tremblay, 1991; Boraud et al., 1998) that may be partially explained by the presence of tremor-related activity (Bergman et al., 1994; Wichmann et al., 1999).

It has been proposed that a breakdown of the functional segregation between parallel re-entrant subcircuits of the basal ganglia-thalamocortical system (Alexander and Crutcher, 1990; Alexander et al., 1990; Hoover and Strick, 1993) might underlie parkinsonian pathophysiology (Filion et al., 1994; Nini et al., 1995; Bergman et al., 1998a). Consistent with this hypothesis, simultaneous microelectrode recording techniques have demonstrated that periodic or "oscillatory" bursting in GPi neurons of

\footnotetext{
Received June 15, 2001; revised Dec. 26, 2001; accepted Jan. 3, 2002.

Funding was provided by the National Institutes of Health, the Canadian Institute of Health Research, and the Parkinson's Foundation of Canada. A.M.L. is a Canadian Institute of Health Research clinician scientist.

Correspondence should be addressed to Jonathan O. Dostrovsky, Department of Physiology, Room 3305, Medical Sciences Building, 1 King's College Circle, University of Toronto, Toronto, Ontario, Canada M5S 1A8. E-mail: j.dostrovsky@utoronto.ca.

Copyright (C) 2002 Society for Neuroscience 0270-6474/02/222855-07\$15.00/0
}

tremulous MPTP-treated monkeys and patients with PD is synchronized (Nini et al., 1995; Bergman et al., 1998b; Hurtado et al., 1999; Raz et al., 2000). We recently reported similar findings in the subthalamic nucleus (STN) of tremulous patients with PD (Levy et al., 2000). These observations support the hypothesis that synchronized oscillations are related to the pathogenesis of limb tremor (Bergman et al., 1998a; Deuschl et al., 2000). It is unclear, however, whether synchronized oscillatory activity is attributable to "direct" synaptic connection or common collateral afferent input within the basal ganglia or simply attributable to synchronized activity occurring elsewhere (such as proprioceptive input or motor cortex-related activity). If synchronization is caused by changes in synaptic efficacy within the basal ganglia, it is possible that the synchronization of neuronal activity is present in patients without limb tremor. To test this hypothesis, we assessed the degree of correlation between the discharge of pairs of neurons in the GPi, GPe, and SNr in tremulous and nontremulous patients with Parkinson's disease.

\section{MATERIALS AND METHODS}

Neurons were recorded during microelectrode-guided placement of a unilateral pallidotomy (eight patients) or bilateral deep brain stimulation (DBS) electrodes in the STN (four patients). The indications for pallidotomy were akinetic-rigid parkinsonian syndromes with fluctuating on-off periods and drug-induced dyskinesias (Lozano et al., 1996). Similarly, the indications for STN DBS were bilateral limb and axial manifestations of PD, medication-refractory motor fluctuations, and drug-induced dyskinesias (Kumar et al., 1998). All patients were studied during the stereotaxic surgery $12-14 \mathrm{hr}$ after the last dose of antiparkinsonian medication, and all were typically akinetic/rigid, with some patients displaying limb tremor (see Results). The patients had a mean age of 59 years (range, 49-69 years), and the average duration of the 

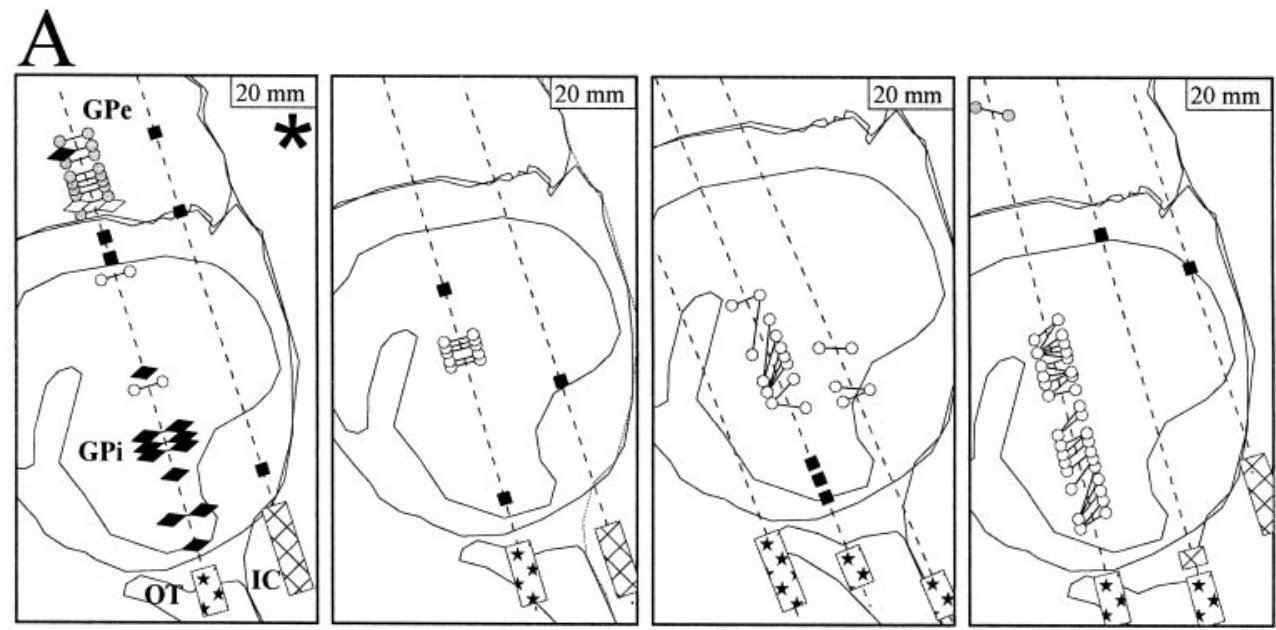

B
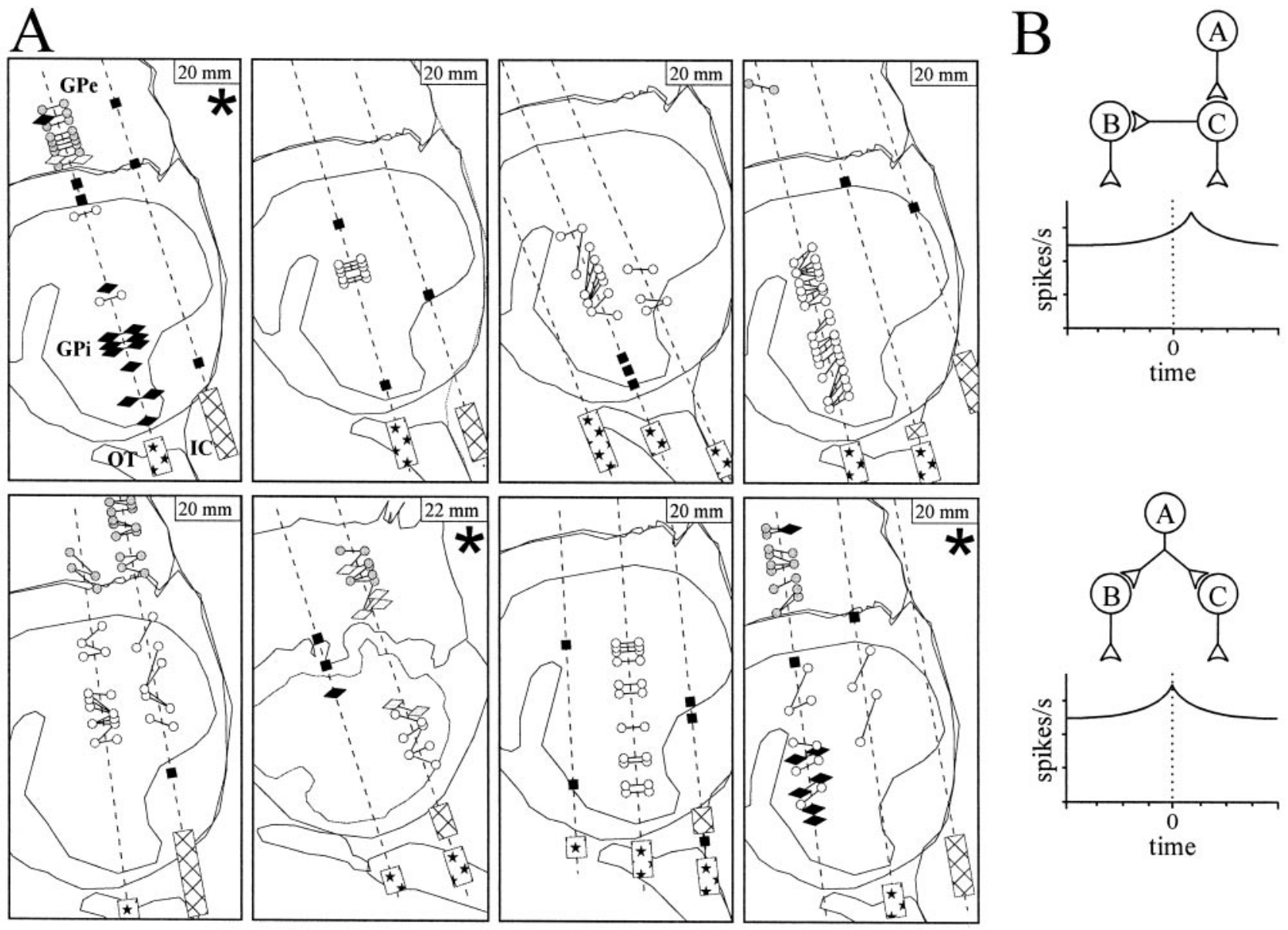
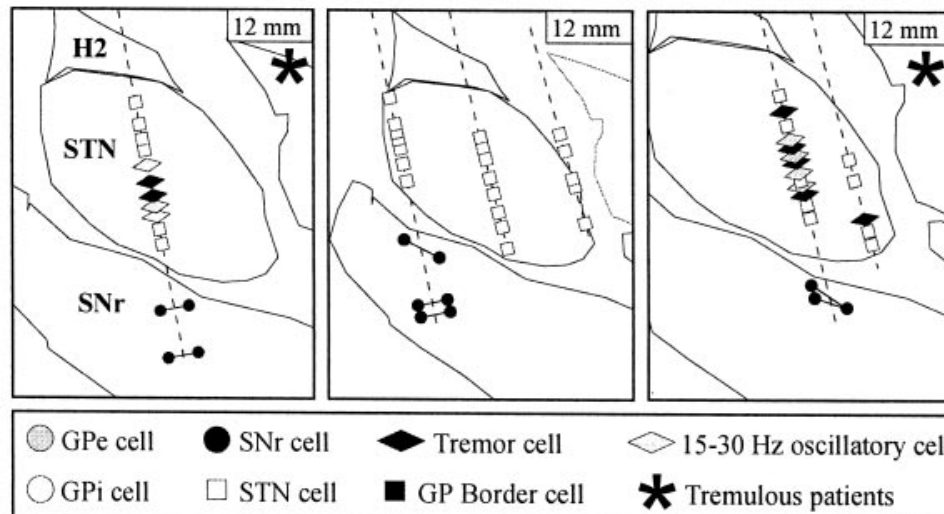

- Tremor cell $\diamond 15-30 \mathrm{~Hz}$ oscillatory cell

- GP Border cell

* Tremulous patients
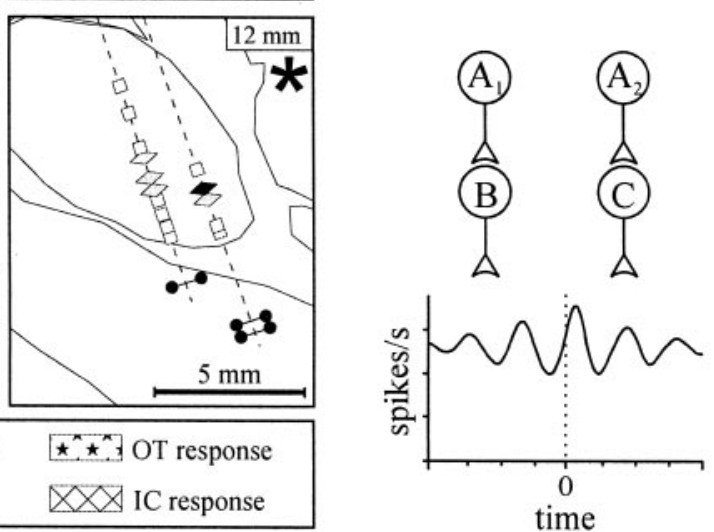

Figure 1. Locations of recorded neurons and the interpretations of the neuron pair cross-correlations. $A$, Sagittal sections based on the Schaltenbrand and Wahren (1977) stereotactic atlas (standardized to the patient's anterior-posterior commissural distance) displaying the microelectrode trajectories (dashed lines) and neuronal activity in the 12 patients (definitions of the symbols used are given at the bottom). The distance from the midline is shown in the upper right corner of each panel. The horizontal distance separating neurons in pairs recorded in the same trajectory has been slightly expanded for clarity (actually $\sim 600 \mu \mathrm{m}$; see Materials and Methods). Optic tract $(O T)$ and internal capsule $(I C)$ responses were obtained using microstimulation. Simultaneously recorded pairs of neurons are joined by lines. H2, Fields of Forel. $B$, Schematic of functional connections between neuron $A$ and a pair of recorded neurons $(B, C)$ that may give rise to synchronization. (Correlograms that would indicate various neuronal connectivity are shown below, with neuron $C$ as the trigger and $A$ as the presynaptic source.) Top, Direct synaptic connection between a neuron pair. Middle, Neurons can be synchronized if they receive common collateral input from a presynaptic source. Bottom, Neurons can display oscillatory synchronization if they receive a common rhythmic drive (i.e., from $A_{1}$ and $A_{2}$ ).

disease was 14 years (range, 7-20 years) at the time of operation. All patients gave free and informed consent, and procedures conformed to guidelines set down by Canadian Institute of Health Research policy on Ethical Conduct for Research Involving Humans and approved by the University of Toronto Ethics Review Board.

Detailed descriptions of the use of microelectrode recording to localize the GPi or the STN have been given previously (Lozano et al., 1996;
Hutchison et al., 1998). Briefly, single-unit microelectrode recordings were used to identify cell types, and stimulation mapping allowed the localization of physiological landmarks (Fig. 1 $A$ ). Single units were recorded using Parylene-coated tungsten microelectrodes with an exposed tip size of $15-25 \mu \mathrm{m}$. Microelectrode tips were plated with gold and platinum to reduce the impedance to $\sim 0.2 \mathrm{M} \Omega$ at $1 \mathrm{kHz}$. The simultaneous recording of neuron pairs was performed using a double- 
electrode setup in which two microelectrodes were inserted either as a glued pair separated by a distance of 250-300 $\mu \mathrm{m}$ or independently, with the electrodes separated by $600 \mu \mathrm{m}$ and each electrode driven by a separate microdrive. All neurons were sampled with the patients at rest. Signals were amplified and filtered using the Guideline System GS3000 (Axon Instruments, Foster City, CA). The two channels of neuronal data were recorded along with wrist flexor/extensor electromyography and accelerometer signals on analog videotape (VR-100 digital recorder; Instrutech Corp., Port Washington, NY) to be analyzed off-line. Singleunit event times were discriminated using template-matching, spikesorting software (Spike2; Cambridge Electronic Design, Cambridge, $\mathrm{UK})$. In this study, the mean number of action potentials recorded per cell was $6776( \pm 530 \mathrm{SEM})$ over an average sample time of $73 \mathrm{sec}( \pm 4.3$ SEM). Only well isolated single neurons recorded from different electrodes were examined.

To compare neuronal activity in this patient group with other patient groups in previous studies, both firing rate and pattern were quantified. Firing pattern was quantified by using the Poisson surprise method of burst detection as described by Legendy and Salcman (1985), which defines a "burst" as an improbable epoch of elevated discharge rate in a spike train. This method was used to detect burst discharges with a Poisson surprise value of $>5$. The number of spikes in burst discharges was compared with the total number of spikes sampled in each cell, and the percentage of spikes in bursts was calculated (Wichmann et al., 1999). This gave a measure of the "burstiness" of each neuron, because irregularly discharging neurons will have a greater proportion of spikes that participate in bursts than regularly discharging neurons.

Autocorrelation and cross-correlation analysis was used to detect oscillatory neuronal activity of single neurons (Karmon and Bergman, 1993) and synchronization between two neurons, respectively. Correlations between separate spike trains can arise from functional connections between pairs of neurons that include direct synaptic connections (Fig. $1 B$, top) or "indirect" connections, such as common collateral input from presynaptic sources (Fig. $1 B$, middle). These functional connections can be detected from the presence of a single asymmetrical or symmetrical peak or trough, respectively, in the cross-correlogram (Moore et al., 1970). In addition, oscillatory synchronization (Fig. 1B, bottom), which arises from synchronous periodic bursting, shows up as multiple peaks and troughs in the cross-correlogram and need not arise from direct or indirect connections between neurons.

Correlograms were calculated for $\pm 1 \mathrm{sec}$ offset ( $5 \mathrm{msec}$ bin size) and $\pm 100 \mathrm{msec}$ offset $(0.5 \mathrm{msec}$ bin size $)$ and were quantified to the units of rate (spikes per second) (Abeles, 1982). To assess synchronization, peaks or troughs in the cross-correlograms were considered significant if they consisted of three or more consecutive bins with values outside a $99 \%$ confidence interval about the mean firing rate (Abeles, 1982). The confidence interval was calculated using correlogram estimates located in the first and last 250 or $25 \mathrm{msec}$ for correlograms constructed with 5 or $0.5 \mathrm{msec}$ bin size, respectively. Oscillatory synchronization was also analyzed using coherence analysis and has been described in detail previously (Levy et al., 2000). Coherence is a function of frequency and is calculated from the cross-spectral density between the two waveforms normalized by the power spectral density of each waveform. Coherence values can range from 0 if the spike trains are not linearly related to a value of 1 if the spike trains have a perfectly linear relationship.

\section{RESULTS}

There were 148 pairs of neurons recorded. Figure $1 A$ displays digitized parasagittal plates (Schaltenbrand and Wahren, 1977) of the GP (Fig. $1 A$, top two rows) and the $\mathrm{SNr}$ (Fig. $1 A$, bottom row) to show the location of the microelectrode trajectories and to provide information about the neuronal activity and the locations of the simultaneously recorded neuron pairs in each patient. Three of eight pallidotomy patients and three of four STN DBS patients had limb tremor at the time of the recordings, and oscillatory neuronal activity was limited to these patients (Fig. $1 A$, asterisk). Examples of raw spike trains simultaneously recorded from pairs of GPi neurons are shown in Figure $2 A$. In the bottom pair, it can be observed that the bursts of spikes are periodic $(\sim 5 \mathrm{~Hz})$ and that the neurons display oscillatory synchronization. The mean firing rate and percentage of spikes in bursts of single neurons in each nucleus are given in Figure $2 B$.
The mean firing rates of neurons in these nuclei were similar to those reported in other patient groups (Hutchison et al., 1994, 1998). The percentage of spikes in bursts in the GPi was also similar to that reported in another patient group (Levy et al., 2001) and was significantly lower than the percentage of spikes in bursts found in the GPe.

\section{Neuronal activity in nontremulous patients or SNr is not correlated}

In the five nontremulous pallidotomy patients, single GP neurons did not display oscillatory activity, and all of the crosscorrelograms in the GPe (15 pairs) and GPi (73 pairs) were flat. An example of cross-correlograms from 20 pairs of GPi neurons recorded from a single nontremulous patient is displayed in Figure $2 C$. (This is the patient whose electrode tracks are shown at the top right of Fig. $1 A$; the cross correlograms are scaled to their confidence intervals.) None of the single $\mathrm{SNr}$ neurons (which were recorded in four patients) displayed oscillations, and synchronized activity was not observed in any of the 10 pairs of $\mathrm{SNr}$ neurons examined. As indicated in Figure $1 A$, three of the four STN/SNr patients had limb tremor during the stereotactic procedure, and many STN neurons displayed oscillatory activity at tremor frequency or in the range of $15-30 \mathrm{~Hz}$. As reported in a previous study, oscillatory activity in the STN occurring in either frequency range was synchronized, but only $15-30 \mathrm{~Hz}$ oscillations were consistently in phase (Levy et al., 2000).

\section{Synchronized tremor-frequency oscillations in tremulous patients}

In the three pallidotomy patients with limb tremor during the microelectrode recordings, some pairs of neurons revealed nonflat cross-correlograms, although the majority of the crosscorrelograms (23 of 29 pairs in the GPe and 15 of 21 pairs in GPi) were also flat. Twelve of $41 \mathrm{GPi}$ and 2 of $47 \mathrm{GPe}$ single neurons (that were sampled from pairs) displayed oscillations in the tremor frequency range $(3-7 \mathrm{~Hz})$. An example of a single GPi neuron that showed a strong coherence with resting tremor of the hand (as measured with an accelerometer) is shown in Figure $3 A$. Coherence between the GPi neuron firing and the limb movement was limited to the period of time with limb tremor. All nonflat cross-correlograms between GP neuron pairs in tremulous patients were oscillatory (with the exception of one pair; see below). Coherence analysis revealed that five of six pairs of GPi tremor cells (TCs) displayed synchronization in the tremor frequency range. Neurons in each of these pairs were located 250 $1000 \mu \mathrm{m}$ from one another. Two examples of tremor-related coherence are displayed in Figure $3 B$. (These are from the patient whose electrode tracks are shown at the top left of Fig. 1A.) Variable phase relationships (i.e., $0-180^{\circ}$ ) were observed between neurons with tremor frequency synchronization.

\section{Synchronized high-frequency oscillations in tremulous patients}

High-frequency oscillatory activity was detected in two of the pallidotomy patients with limb tremor. In one of these patients, these neurons were recorded simultaneously, and their activity was correlated. It was found that one pair of GPi neurons and five pairs of GPe neurons displayed coherent high-frequency oscillatory activity. The mean frequency of the oscillatory synchronization was $20.6 \mathrm{~Hz}$ (range 19-22), with a mean absolute phase difference of $16.0^{\circ}$ (range $3-35^{\circ}$ ). Examples of high-frequency coherence in a pair of GPe and GPi neurons are shown in Figure $3 C$. In the same patient, one pair of GPe neurons displayed an 

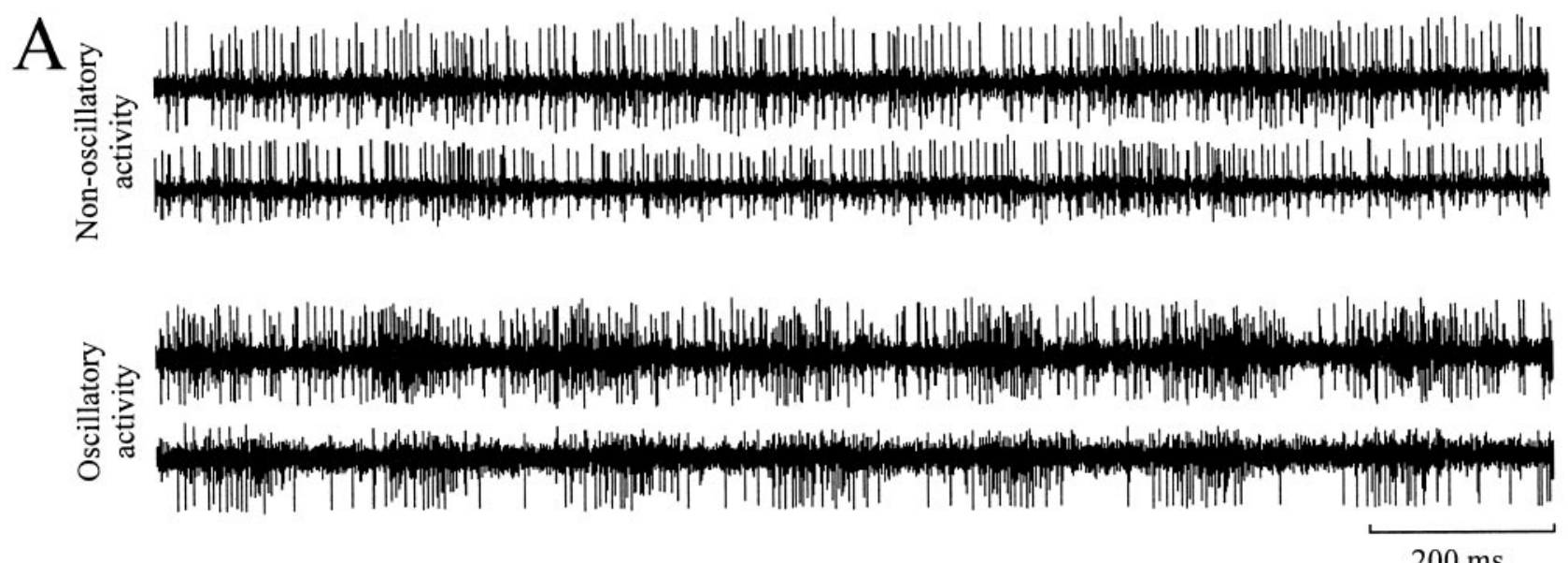

B
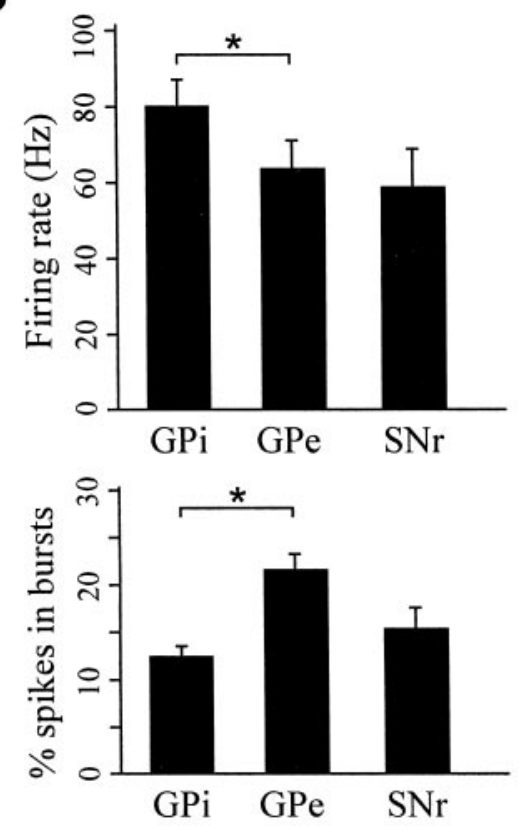

C
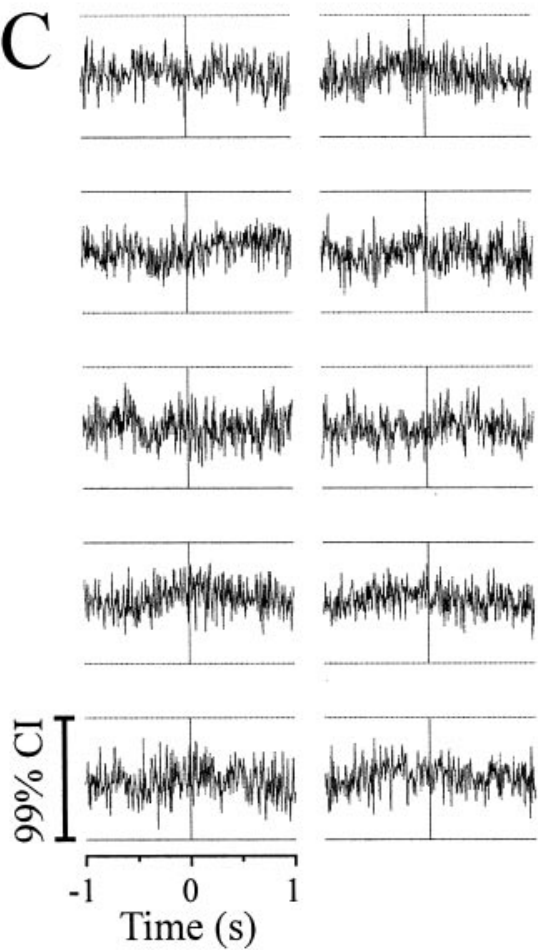
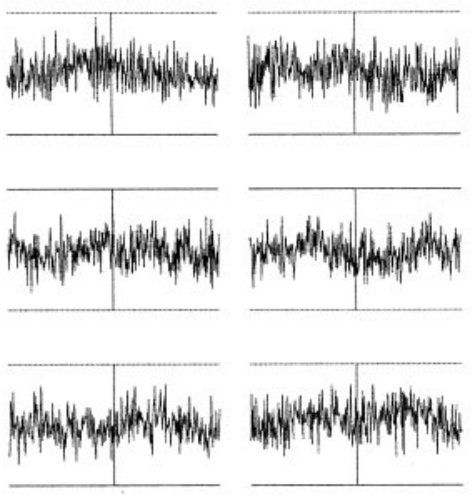

$200 \mathrm{~ms}$
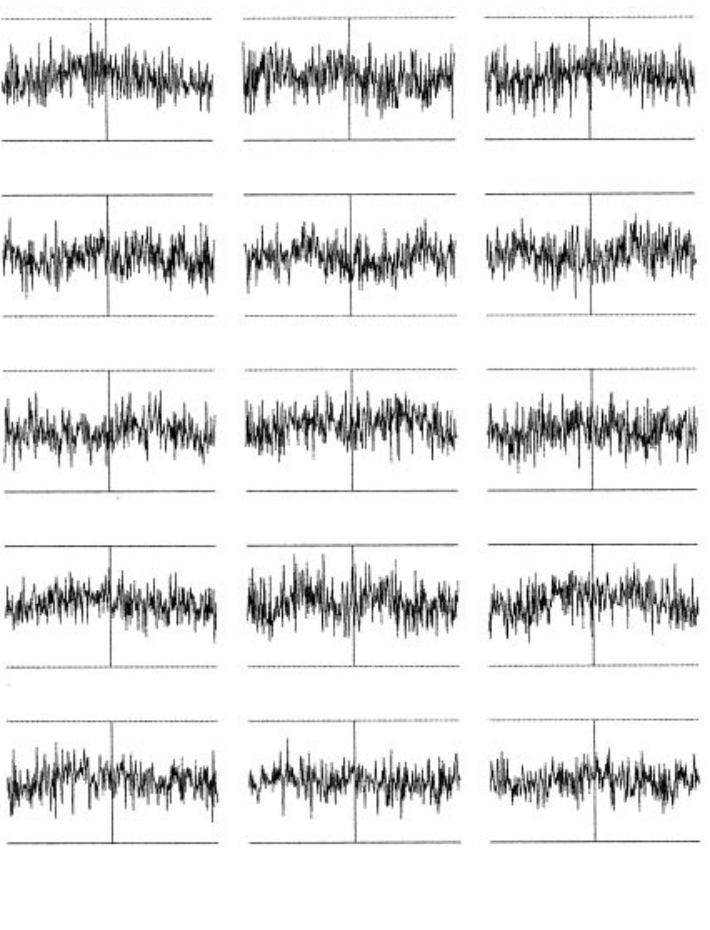

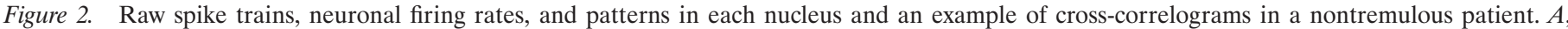

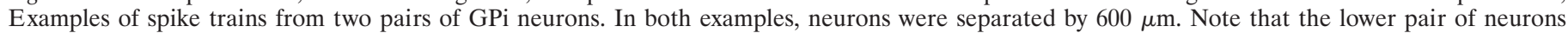

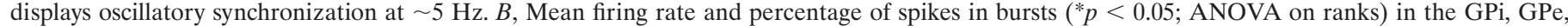

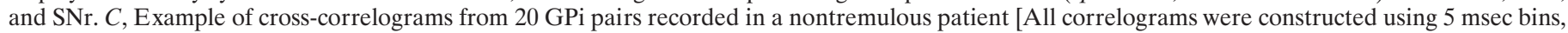
normalized to baseline firing rate and scaled to the $99 \%$ confidence interval $(C I)$.]

asymmetrical trough in the cross-correlogram that was centered at $\sim 2 \mathrm{msec}$, most likely indicating a direct inhibitory connection from one neuron to the other (Fig. 3D) (Perkel et al., 1967).

\section{DISCUSSION}

The loss-of-segregation hypothesis predicts that dopamine depletion in PD results in a breakdown of the independent activity in subcircuits of the basal ganglia (Filion et al., 1994; Nini et al., 1995; Bergman et al., 1998a; Vitek and Giroux, 2000). This study and our data from the STN (Levy et al., 2000) demonstrate that in parkinsonian patients, discharge synchronization between pairs of neurons in the GPi, GPe, and STN is limited to oscillatory activity in patients with limb tremor and is indicative of common rhythmic drive to neuron pairs rather than to direct interaction between pairs of neurons. In all nontremulous patients examined, synchronization was not observed, suggesting that neuron pair synchronization attributable to direct synaptic connections or common input is not present in the parkinsonian basal ganglia. These conclusions are consistent with the findings of studies in monkeys that examined the simultaneous activity of pairs of GPi, GPe, STN, and striatal tonically active neurons and failed to find an increase in nonoscillatory synchronization after MPTP-induced parkinsonism (Bergman et al., 1994; Nini et al., 1995; Raz et al., 1996, 2000, 2001).

Evidence of a direct functional connection (inhibitory synaptic connection) was only observed in 1 of 44 pairs of GPe neurons 
A
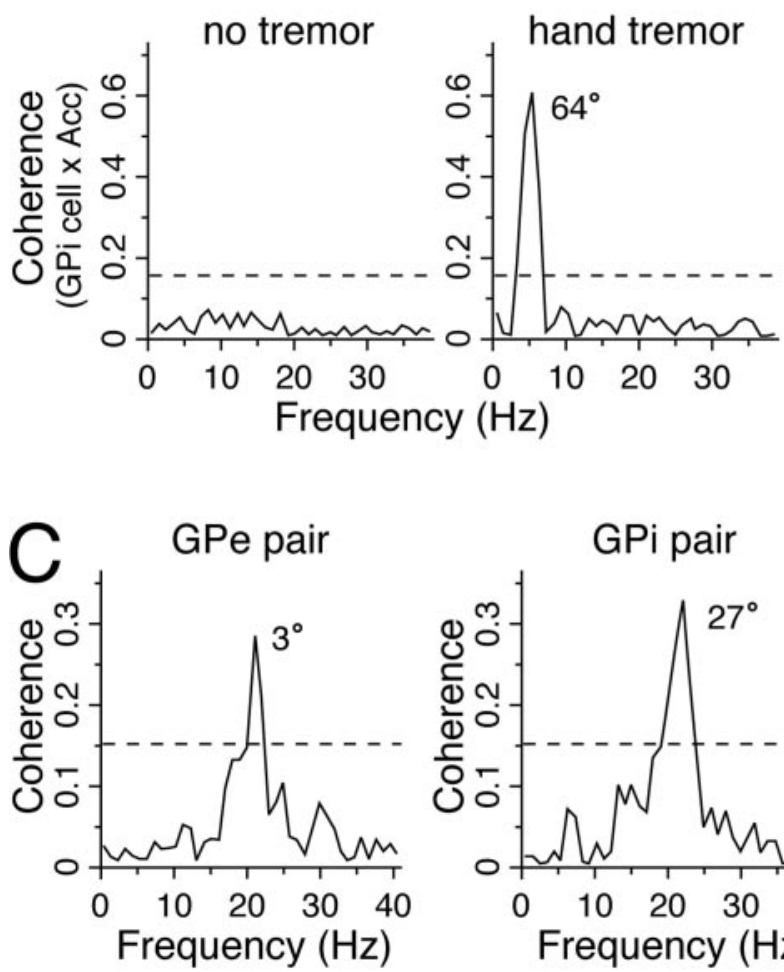
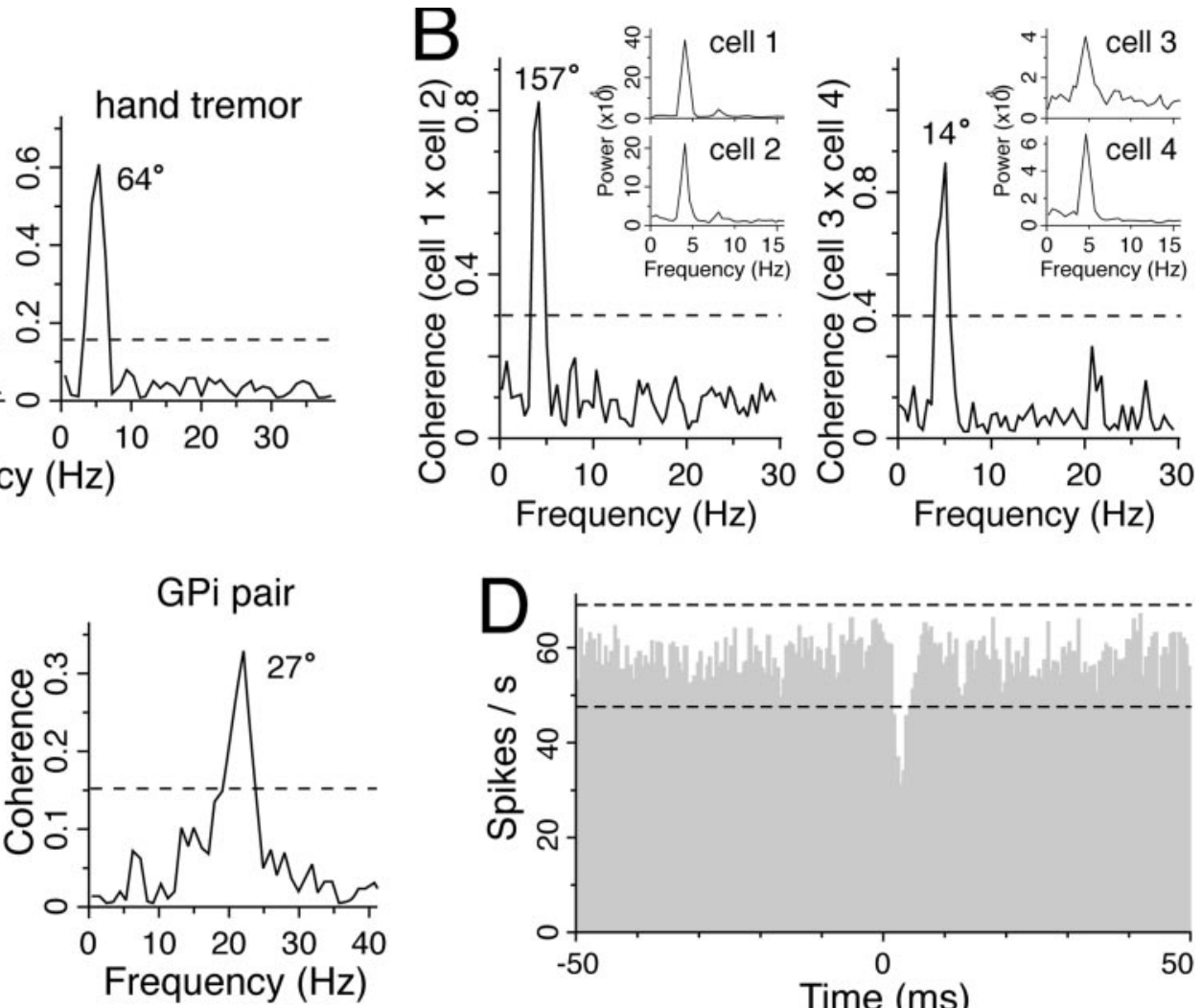

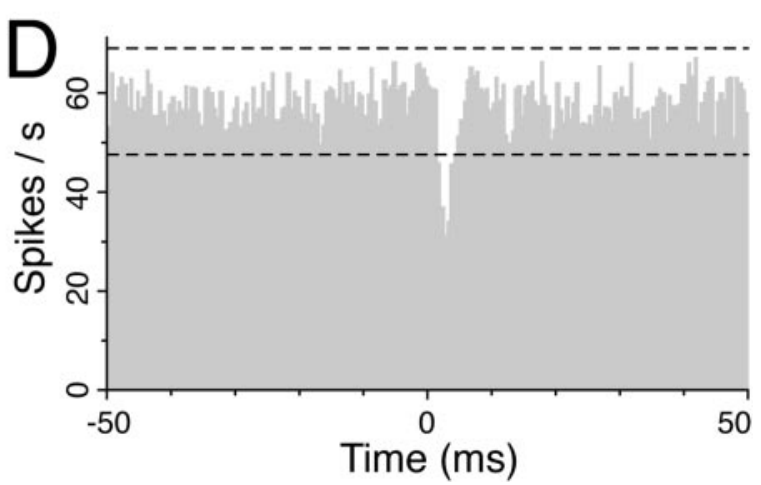

Figure 3. Synchronized activities between pairs of neurons in tremulous patients. $A$, Example of coherence between contralateral hand tremor [measured with an accelerometer $(A c c)$ ] and GPi cell activity. Left, Coherence during $30 \mathrm{sec}$ with no tremor. Right, Coherence during a subsequent 30 sec with limb tremor $(0.98 \mathrm{~Hz}$ resolution; the number beside the peak is the phase difference in degrees, and the dashed horizontal line is $p<0.01)$. $B$, Example of power spectra (insets) and coherence of two pairs of GPi TCs from a single patient ( $0.49 \mathrm{~Hz}$ resolution, calculated using 30 sec of data for each pair; the number beside the peak is the phase difference in degrees, and the dashed horizontal line is $p<0.01$ for coherence). These plots demonstrate tremor-related synchronization and phase variability in the GPi of a tremulous patient. Neurons in each pair were separated by $250 \mu \mathrm{m}$, and pairs were located $\sim 200 \mu \mathrm{m}$ from one another. C, Examples of coherence plots showing high-frequency oscillatory synchronization in the GPe (left) and GPi (right) of a tremulous pallidotomy patient (calculated from $30 \mathrm{sec}$ of data, $0.98 \mathrm{~Hz}$ resolution; the number beside the peak is the phase difference in degrees, and the dashed horizontal line is $p<0.01)$. D, A nonflat cross-correlogram in a tremulous pallidotomy patient that is indicative of an inhibitory connection between a pair of GPe neurons $(0.5 \mathrm{msec}$ bins; $7010 \times 9252$ events; horizontal dashed lines indicate the $99 \%$ confidence interval about the mean).

(Fig. 3D). This observation is consistent with a recent study by Raz et al. (2000) in intact monkeys in which $\sim 1 \%$ of the crosscorrelograms of GPe neurons displayed a significant trough. It has been observed that (GABAergic) GPe neurons emit short, intranuclear axon collaterals (Oertel et al., 1984; Smith et al., 1987; Sato et al., 2000) and could account for the observed inhibition in the cross-correlogram. We did not encounter SNr neurons with oscillatory activity in this study, although three of four patients displayed limb tremor and tremor-related oscillations were observed in the STN. This is consistent with the observation in MPTP-treated monkeys and parkinsonian patients that tremorrelated activity is not as prominent in the $\mathrm{SNr}$ as it is in the GPi or STN (Wichmann et al., 1999; Rodriguez-Oroz et al., 2001). It is possible, however, that differences in sampling (i.e., motorrelated neurons in the GPi vs non-motor-related neurons in the $\mathrm{SNr}$ ) in parkinsonian patients might account for the lack of tremor-related oscillatory activity in the SNr. The SNr neurons sampled in this study may not have been in the motor area of the $\mathrm{SNr}$, because this region is believed to be in the more intermediate and lateral portion of the nucleus (DeLong et al., 1983; Schultz, 1986; Hutchison et al., 1998). It has also been suggested that the GPi contains a greater proportion of neurons with motorrelated activity than the $\mathrm{SNr}$; therefore, the changes in discharge activity attributed to parkinsonism may be greater in the GPi than the SNr (Wichmann et al., 1999).

The main limitation of this study is the possibility that crosscorrelation between pairs of neurons cannot detect weak neuronal synchronization that might involve a larger population of neurons. For example, Brown et al. (2001) demonstrated that a 15-30 Hz synchronization between the GPi and STN could be detected in parkinsonian patients by recording local field potentials in these nuclei. This synchronization is reduced by the administration of 3,4-dihydroxyphenylalanine (L-dopa) and most likely plays a role in the pathophysiology of the akinesia and bradykinesia (Marsden et al., 2001). It is also reasonable to suppose that a longer sampling time may detect weaker synchronization between pairs of neurons. It should be noted, however, that with similar sampling times, a substantially greater amount of nonoscillatory synchronization has been reported in the thalamus in humans (Levy et al., 1999) and in the cortex in monkeys (Nini et al., 1995). Another drawback of cross-correlation analysis is that it is less sensitive for inhibitory than for excitatory functional connectivity (of comparable strength), and therefore, it is possible that some neuron-to-neuron inhibition would have failed to be detected using this technique (Aertsen and Gerstein, 1985). In addition, an obvious difference between studies comparing syn- 
chronization in MPTP-treated monkeys and patients with PD is that patients undergo chronic L-dopa therapy over a number of years and develop drug-induced dyskinesias (excessive abnormal involuntary movements), whereas parkinsonian monkeys are not similarly treated with dopaminergic medication. Therefore, it is possible that the MPTP monkey model does not replicate the idiopathic PD (observed after years of dopaminergic treatment) in all its physiological underpinnings. Finally, it is feasible that overt neuronal synchronization in parkinsonism is displayed during active or passive movements. It has been shown that after MPTP treatment in monkeys, there is an increase in the number of GPi neurons responding to passive movement and neurons tend to display exaggerated responses and multilimb receptive fields (Filion et al., 1988; Boraud et al., 2000). The administration of apomorphine, a nonselective $\mathrm{D}_{1} / \mathrm{D}_{2}$ receptor agonist, decreases the proportion of neurons with multilimbed receptive fields in parkinsonian patients (Levy et al., 2001). However, preliminary data from our group that examine the simultaneous activity of pairs of neurons during a reaching task do not demonstrate that neurons with similar responses have correlated discharges indicative of underlying synaptic connections.

Our results of GPi TC synchronization are consistent with a previous report in a single tremulous parkinsonian patient and in studies in MPTP-treated monkeys with resting tremor and/or postural/action tremor (vervet vs rhesus monkeys) (Nini et al., 1995; Bergman et al., 1998b; Hurtado et al., 1999; Raz et al., 2000). Similar to activity in the STN (Levy et al., 2000), tremor frequency oscillations in the GPi can be synchronized and have variable phase relationships. This study also described the novel finding of in-phase 15-30 Hz ("high-frequency") neuronal oscillatory synchronization in the pallidum of a patient with tremor and supports microelectrode findings in MPTP-treated vervet monkeys (Bergman et al., 1998b) and field potential analysis in parkinsonian patients (Brown et al., 2001). Our observations in the GPe are consistent with the demonstration in tremulous MPTP-treated monkeys that pairs of GPe cells exhibit highfrequency oscillatory synchronization with phase differences that are centered around $0^{\circ}$ (Raz et al., 2000), similar to our initial findings in the STN of tremulous parkinsonian patients (Levy et al., 2000). It is likely that pallidal high-frequency oscillations are secondary to high-frequency oscillations transmitted via the cortico-STN pathway (Marsden et al., 2001). These observations suggest that the GPe-STN network (Plenz and Kital, 1999; Magill et al., 2000) might be involved in maintaining in-phase high-frequency oscillatory synchronization in patients with limb tremor (Levy et al., 2000).

In summary, this study demonstrates that the synchronization of neuron pairs in the GP is limited to oscillatory activity occurring in the tremor frequency range and in the $15-30 \mathrm{~Hz}$ range. The lack of correlated activity in patients without limb tremor suggests that significant neuron pair synchronization caused by direct synaptic connections or common input is not present in the parkinsonian basal ganglia.

\section{REFERENCES}

Abeles M (1982) Quantification, smoothing, and confidence limits for single-units' histograms. J Neurosci Methods 5:317-325.

Aertsen AM, Gerstein GL (1985) Evaluation of neuronal connectivity: sensitivity of cross-correlation. Brain Res 340:341-354.

Albin RL, Young AB, Penney JB (1989) The functional anatomy of basal ganglia disorders. Trends Neurosci 12:366-375.

Alexander GE, Crutcher MD (1990) Functional architecture of basal ganglia circuits: neural substrates of parallel processing. Trends Neurosci 13:266-271.
Alexander GE, Crutcher MD, DeLong MR (1990) Basal gangliathalamocortical circuits: parallel substrates for motor, oculomotor, "prefrontal" and "limbic" functions. Prog Brain Res 85:119-146.

Bergman H, Wichmann T, Karmon B, DeLong MR (1994) The primate subthalamic nucleus. II. Neuronal activity in the MPTP model of parkinsonism. J Neurophysiol 72:507-520.

Bergman H, Feingold A, Nini A, Raz A, Slovin H, Abeles M, Vaadia E (1998a) Physiological aspects of information processing in the basal ganglia of normal and parkinsonian primates. Trends Neurosci 21:32-38.

Bergman H, Raz A, Feingold A, Nini A, Nelken I, Hansel D, Ben Pazi H, Reches A (1998b) Physiology of MPTP tremor. Mov Disord 13 [Suppl 3]:29-34.

Boraud T, Bezard E, Guehl D, Bioulac B, Gross C (1998) Effects of L-DOPA on neuronal activity of the globus pallidus externalis (GPe) and globus pallidus internalis (GPi) in the MPTP-treated monkey. Brain Res 787:157-160.

Boraud T, Bezard E, Bioulac B, Gross CE (2000) Ratio of inhibited-toactivated pallidal neurons decreases dramatically during passive limb movement in the MPTP-treated monkey. J Neurophysiol 83:1760-1763.

Brown P, Oliviero A, Mazzone P, Insola A, Tonali P, Di Lazzaro V (2001) Dopamine dependency of oscillations between subthalamic nucleus and pallidum in Parkinson's disease. J Neurosci 21:1033-1038.

Crossman AR, Mitchell IJ, Sambrook MA (1985) Regional brain uptake of 2-deoxyglucose in $N$-methyl-4-phenyl-1,2,3,6-tetrahydropyridine (MPTP)-induced parkinsonism in the macaque monkey. Neuropharmacology 24:587-591.

DeLong MR (1990) Primate models of movement disorders of basal ganglia origin. Trends Neurosci 13:281-285.

DeLong MR, Crutcher MD, Georgopoulos AP (1983) Relations between movement and single cell discharge in the substantia nigra of the behaving monkey. J Neurosci 3:1599-1606.

Deuschl G, Raethjen J, Baron R, Lindemann M, Wilms H, Krack P (2000) The pathophysiology of parkinsonian tremor: a review. J Neurol 247 [Suppl 5]:V33-V48.

Eidelberg D, Moeller JR, Kazumata K, Antonini A, Sterio D, Dhawan V, Spetsieris P, Alterman R, Kelly PJ, Dogali M, Fazzini E, Beric A (1997) Metabolic correlates of pallidal neuronal activity in Parkinson's disease. Brain 120:1315-1324.

Filion M, Tremblay L (1991) Abnormal spontaneous activity of globus pallidus neurons in monkeys with MPTP-induced parkinsonism. Brain Res 547:142-151.

Filion M, Tremblay L, Bedard PJ (1988) Abnormal influences of passive limb movement on the activity of globus pallidus neurons in parkinsonian monkeys. Brain Res 444:165-176.

Filion M, Tremblay L, Matsumura M, Richard H (1994) Dynamic focusing of informational convergence in basal ganglia. Rev Neurol (Paris) 150:627-633.

Hoover JE, Strick PL (1993) Multiple output channels in the basal ganglia. Science 259:819-821.

Hurtado JM, Gray CM, Tamas LB, Sigvardt KA (1999) Dynamics of tremor-related oscillations in the human globus pallidus: a single case study. Proc Natl Acad Sci USA 96:1674-1679.

Hutchison WD, Lozano AM, Davis KD, Saint-Cyr JA, Lang AE, Dostrovsky JO (1994) Differential neuronal activity in segments of globus pallidus in Parkinson's disease patients. NeuroReport 5:1533-1537.

Hutchison WD, Allan RJ, Opitz H, Levy R, Dostrovsky JO, Lang AE, Lozano AM (1998) Neurophysiological identification of the subthalamic nucleus in surgery for Parkinson's disease. Ann Neurol 44:622-628.

Karmon B, Bergman H (1993) Detection of neuronal periodic oscillations in the basal ganglia of normal and parkinsonian monkeys. Isr J Med Sci 29:570-579.

Kumar R, Lozano AM, Kim YJ, Hutchison WD, Sime E, Halket E, Lang AE (1998) Double-blind evaluation of subthalamic nucleus deep brain stimulation in advanced Parkinson's disease. Neurology 51:850-855.

Lang AE, Lozano AM (1998) Parkinson's disease. First of two parts. N Engl J Med 339:1044-1053.

Legendy CR, Salcman M (1985) Bursts and recurrences of bursts in the spike trains of spontaneously active striate cortex neurons. J Neurophysiol 53:926-939.

Levy R, Davis KD, Hutchison WD, Pahapill PA, Lozano AM, Tasker RR, Dostrovsky JO (1999) Simultaneously recorded neuron pairs in the motor thalamus of patients with Parkinson's disease and essential tremor. Soc Neurosci Abstr 25:1408.

Levy R, Hutchison WD, Lozano AM, Dostrovsky JO (2000) Highfrequency synchronization of neuronal activity in the subthalamic nucleus of parkinsonian patients with limb tremor. $J$ Neurosci 20:7766-7775.

Levy R, Dostrovsky JO, Lang AE, Sime E, Hutchison WD, Lozano AM (2001) Effects of apomorphine on subthalamic nucleus and globus pallidus internus neurons in patients with Parkinson's disease. J Neurophysiol 86:249-260. 
Lozano A, Hutchison W, Kiss Z, Tasker R, Davis K, Dostrovsky J (1996) Methods for microelectrode-guided posteroventral pallidotomy. J Neurosurg 84:194-202.

Magill PJ, Bolam JP, Bevan MD (2000) Relationship of activity in the subthalamic nucleus-globus pallidus network to cortical electroencephalogram. J Neurosci 20:820-833.

Marsden JF, Limousin-Dowsey P, Ashby P, Pollak P, Brown P (2001) Subthalamic nucleus, sensorimotor cortex and muscle interrelationships in Parkinson's disease. Brain 124:378-388.

Miller WC, DeLong MR (1987) Altered tonic activity of neurons in the globus pallidus and subthalamic nucleus in the primate MPTP model of parkinsonism. In: The basal ganglia. II. Structure and function: current concepts (Carpenter MB, Jayaraman A, eds), pp 415-427. New York: Plenum.

Moore GP, Segundo JP, Perkel DH, Levitan H (1970) Statistical signs of synaptic interaction in neurons. Biophys J 10:876-900.

Nini A, Feingold A, Slovin H, Bergman H (1995) Neurons in the globus pallidus do not show correlated activity in the normal monkey, but phase-locked oscillations appear in the MPTP model of parkinsonism. J Neurophysiol 74:1800-1805.

Oertel WH, Nitsch C, Mugnaini E (1984) Immunocytochemical demonstration of the GABA-ergic neurons in rat globus pallidus and nucleus entopeduncularis and their GABA-ergic innervation. Adv Neurol 40:91-98.

Perkel DH, Gerstein GL, Moore GP (1967) Neuronal spike trains and stochastic point processes. II. Simultaneous spike trains. Biophys J 7:419-440.

Plenz D, Kital ST (1999) A basal ganglia pacemaker formed by the subthalamic nucleus and external globus pallidus. Nature 400:677-682.

Raz A, Feingold A, Zelanskaya V, Vaadia E, Bergman H (1996) Neu- ronal synchronization of tonically active neurons in the striatum of normal and parkinsonian primates. J Neurophysiol 76:2083-2088.

Raz A, Vaadia E, Bergman H (2000) Firing patterns and correlations of spontaneous discharge of pallidal neurons in the normal and the tremulous 1-methyl-4-phenyl-1,2,3,6-tetrahydropyridine vervet model of parkinsonism. J Neurosci 20:8559-8571.

Raz A, Frechter-Mazar V, Feingold A, Abeles M, Vaadia E, Bergman H (2001) Activity of pallidal and striatal tonically active neurons is correlated in MP TP-treated monkeys but not in normal monkeys. J Neurosci 21:RC128.

Rodriguez-Oroz MC, Rodriguez M, Guridi J, Mewes K, Chockkman V, Vitek J, DeLong MR, Obeso JA (2001) The subthalamic nucleus in Parkinson's disease: somatotopic organization and physiological characteristics. Brain 124:1777-1790.

Sato F, Lavallee P, Levesque M, Parent A (2000) Single-axon tracing study of neurons of the external segment of the globus pallidus in primate. J Comp Neurol 417:17-31.

Schaltenbrand G, Wahren W (1977) Atlas for stereotaxy of the human brain. Stuttgart, Germany: Thieme.

Schultz W (1986) Activity of pars reticulata neurons of monkey substantia nigra in relation to motor, sensory, and complex events. J Neurophysiol 55:660-677.

Smith Y, Parent A, Seguela P, Descarries L (1987) Distribution of GABA-immunoreactive neurons in the basal ganglia of the squirrel monkey (Saimiri sciureus). J Comp Neurol 259:50-64.

Vitek JL, Giroux M (2000) Physiology of hypokinetic and hyperkinetic movement disorders: model for dyskinesia. Ann Neurol 47:S131-S140.

Wichmann T, Bergman H, Starr PA, Subramanian T, Watts RL, DeLong MR (1999) Comparison of MPTP-induced changes in spontaneous neuronal discharge in the internal pallidal segment and in the substantia nigra pars reticulata in primates. Exp Brain Res 125:397-409. 